\title{
Medicinal supplement genipin induces p53 and Bax-dependent apoptosis in colon cancer cells
}

\author{
JINGWANG YE ${ }^{1 *}, \mathrm{JING} \mathrm{LI}^{2 *}$, XIANGFENG WANG ${ }^{1}$ and LING LI ${ }^{3}$ \\ Departments of ${ }^{1}$ Gastrointestinal Surgery, ${ }^{2}$ Central Sterile Supply and ${ }^{3}$ Vasculocardiology, \\ Research Institute of Surgery, Daping Hospital, Third Military Medical University, Chongqing 400042, P.R. China
}

Received October 8, 2017; Accepted May 31, 2018

DOI: $10.3892 /$ ol.2018.9025

\begin{abstract}
Colorectal cancer (CRC) is the third leading cause of cancer-associated mortality worldwide. Genipin is a medicinal herb compound derived from the gardenia fruit, which has been reported to exhibit antitumor activity against several types of cancer. The aim of the present study was to investigate the antitumor effect of genipin on colon cancer and the underlying molecular mechanisms. Genipin significantly inhibited the viability of HCT116 and SW480 cells in vitro in a dose- and time-dependent manner. Additionally, genipin was able to significantly inhibit tumor growth in nude mice with xenografts of HCT116 and SW480 cells. The inhibition of tumor growth by genipin treatment was coupled with G0/G1 cell cycle arrest, apoptosis induction, increased reactive oxygen species damage and loss of mitochondrial membrane potential. Further investigation of genipin-treated HCT116 cells revealed that the expression of p53, Bax and cleaved caspase-3 in genipin-treated cells was increased compared with the vehicle control, whereas B-cell lymphoma-2 expression appeared to be lower in genipin-treated cells. Collectively, the findings of the present study indicate that genipin was able to decrease proliferation and promote apoptosis in colon cancer cells by inducing the p53/Bax-mediated signaling pathway. Therefore, genipin may be used as a novel therapeutic agent in the treatment of CRC.
\end{abstract}

Correspondence to: Dr Xiangfeng Wang, Department of Gastrointestinal Surgery, Research Institute of Surgery, Daping Hospital, Third Military Medical University, 10 Changjiang Branch Road, Chongqing 400042, P.R. China

E-mail: 357579943@qq.com

Miss Ling Li, Department of Vasculocardiology, Research Institute of Surgery, Daping Hospital, Third Military Medical University, 10 Changjiang Branch Road, Chongqing 400042, P.R. China E-mail: lilingdr@126.com

${ }^{*}$ Contributed equally

Key words: apoptosis, genipin, HCT116, G0/G1 arrest, mitochondrial pathway, p53

\section{Introduction}

Colorectal cancer (CRC) currently ranks third among the leading causes of cancer-related mortality worldwide, with $>1.2$ million new cases and >600,000 deaths annually (1). Due to the increasing trend in the number of cases and mortality rate, research has been focused on investigating potentially effective therapeutic compounds that may control the malignant progression of CRC. Due to the attempts of scientists and clinicians to discover therapeutic compounds that effectively treat $\mathrm{CRC}$ while reducing treatment-related toxicity, there has been a resurgence of interest in investigating the antitumor activity of medicinal herbs and dietary compounds $(2,3)$.

Genipin, a medicinal herb compound derived from the gardenia fruit, has been used as a traditional Chinese medicine over several decades, and recent reports have demonstrated that genipin possesses medicinal properties, including anti-inflammatory (4), anti-angiogenic (5) and anti-thrombotic properties (6). Genipin has also exhibited significant anititumor activity against several types of cancer, such as gastric cancer (7), hepatocellular carcinoma (8), breast cancer (9) and cervical cancer (10). However, the effect of genipin on colon cancer has yet to be investigated.

The aim of the present study was to determine the therapeutic effects of genipin on colon cancer cells and elucidate the molecular mechanisms underlying the medicinal properties of genipin in colon cancer, in order to provide the basis for future research on genipin as a pharmaceutical compound in colon cancer treatment.

\section{Materials and methods}

Genipin, cell lines and nude mice. Genipin (98\%) was purchased from Xi'an Kailai Biology Company (Xi'an, China). HCT116 and SW480 cells were purchased from the American Type Culture Collection (Manassas, VA, USA). HCT116 cells were routinely cultured in McCoy's 5A medium supplemented with $10 \%$ fetal calf serum (both Gibco; Thermo Fisher Scientific, Inc., Waltham, MA, USA) and SW480 cells were grown in Dulbecco modified Eagle's medium (DMEM) supplemented with $10 \%$ fetal bovine serum (FBS) and $5 \% 0.1 \mathrm{mM}$ penicillin-streptomycin, and all cells were incubated at $37^{\circ} \mathrm{C}$ in $5 \% \mathrm{CO}_{2}$. Male Balb/c nude mice (180-220 g, 5-6 weeks old) were provided by the Third Military Medical University 
(Chongqing, China) and maintained with standard food and water at $27 \pm 2^{\circ} \mathrm{C}$. The animal experiments conducted in this study were approved by the Laboratory Animal Welfare and Ethics Committee of the Third Military Medical University.

Cell viability assay. HCT116 or SW480 cells were seeded into 96-well plates at a density of 7,000 cells per well and incubated with genipin $(, 150,300,450$ or $600 \mu \mathrm{M})$ for 12,24 and $36 \mathrm{~h}$. After treatment, the culture medium was replaced with $10 \mu \mathrm{l}$ CCK-8 (Dojindo Molecular Technologies, Inc., Kumamoto, Japan) and $90 \mu \mathrm{l}$ medium per well and incubated in the dark for $2 \mathrm{~h}$ at $37^{\circ} \mathrm{C}$. Finally, the optical density (OD) for each well was measured at $450 \mathrm{~nm}$ with a microplate reader.

Cell morphology observation and DAPI staining. HCT116 cells $\left(\sim 5 \times 10^{5}\right)$ were seeded into each well of a 6 -well plate and, following treatment with genipin for $24 \mathrm{~h}$, the cells were fixed with $4 \%$ (v/v) for $10 \mathrm{~min}$ and observed under an inverted light microscope. The cells were then stained with DAPI (Solarbio, Beijing, China) for $20 \mathrm{~min}$, and images were captured with a fluorescence microscope. Image-ProPlus 6.0 software was used to calculate the mean fluorescence intensity of the DAPI-stained cells.

Cell cycle distribution and apoptosis analysis. For cell cycle analysis, genipin-treated HCT116 cells were fixed with pre-cooled $70 \%$ ethanol overnight. Following incubation with RNaseA $(0.25 \mathrm{mg} / \mathrm{ml})$ for $30 \mathrm{~min}$ at $37^{\circ} \mathrm{C}$, the cells were incubated with $20 \mu \mathrm{g} / \mathrm{ml}$ propidium iodide (PI; Solarbio) in the dark for $30 \mathrm{~min}$. Finally, 10,000 cells per sample were analyzed by flow cytometry. FlowJo 7.6 software (FlowJo LLC, Ashland, OR, USA) was used to evaluate the cells at the G1, S and G2 phase. For apoptotic analysis, double staining with an Annexin V (FITC-conjugated) and PI (Solarbio) was used to analyze earlyand late-stage apoptosis. HCT116 cells were washed twice with cold phosphate-buffered saline and suspended in binding buffer with $10^{6}$ cells $/ \mathrm{ml}$. The cells were then stained with Annexin V (FITC-conjugated, $10 \mu \mathrm{l} / \mathrm{ml})$ and PI $(10 \mu \mathrm{l} / \mathrm{ml})$ for $25 \mathrm{~min}$ in the dark prior to being analyzed by flow cytometry.

Determination of mitochondrial membrane potential $(M M P)$ and reactive oxygen species (ROS). To determine the MMP, genipin-treated HCT116 cells were stained with $10 \mu \mathrm{M}$ Rhodamine 123 (Solarbio) for $30 \mathrm{~min}$ and evaluated by fluorescence microscopy. To determine ROS generation, genipin-treated HCT116 cells were stained with $20 \mu \mathrm{M}$ DCFH-DA (Solarbio) for $30 \mathrm{~min}$ and evaluated by flow cytometry.

Western blot analysis. Cells or tissue were lysed in RIPA lysis buffer (Sigma-Aldrich; Merck-KGaA, USA) on ice for $30 \mathrm{~min}$ and subsequently centrifuged to obtain the lysate supernatant. Protein lysates (30-50 $\mu \mathrm{g}$ total protein), were run on an SDS-PAGE gel and electro transferred onto PVDF membranes (Thermo Fisher Scientific, Inc.). Appropriate primary antibodies were added to blocking buffer and incubated with the membrane overnight at $4^{\circ} \mathrm{C}$. After washing, the membranes were incubated with the corresponding secondary antibodies (ZSGB-BIO) for $2 \mathrm{~h}$. Finally, the bands were visualized by the Odyssey Infrared Imaging System (Li-Cor
Biosciences, Lincoln, NE, USA). The antibodies utilized included anti-Bax (dilution, 1:800; ab53154), anti-cleaved caspase-3 (dilution, 1:500; ab2302), anti-p53 (dilution, 1:800; ab131442), anti-B-cell lymphoma (Bcl)-2 (dilution, 1:500; ab182858; all Abcam, Cambridge, UK) and anti- $\beta$-actin (dilution, 1:500; sc-130065; Santa Cruz Biotechnology Inc., Dallas, TX, USA), Goat anti-Rabbit IgG (H+L) Highly Cross-Adsorbed secondary antibodies, Alexa Fluor Plus 800 (dilution, 1:100,000; A32735), Donkey anti-Mouse IgG (H+L) Secondary antibodies, WesternDot 800 (dilution, 1:5,000; W10823, Invitrogen; Thermo Fisher Scientific, Inc.).

Animal experiments. Male Balb/c nude micewere subcutaneously injected with $5 \times 10^{7}$ HCT116 or SW480 cells. After $\sim 3$ weeks, when the subcutaneous tumors had grown to $2 \mathrm{~mm}^{3}$, the xenografted mice were randomly divided into four groups ( $n=5$ per group). In 4 of the groups, the mice were intragastrically administered genipin (dissolved by saline, 20,40 , or $80 \mathrm{mg} / \mathrm{kg}$ per day) for 4 weeks, while the control group mice were gavaged with saline. The diameter of the tumor was measured weekly by a vernier caliper to calculate the tumor volume ( $\mathrm{V}=\mathrm{L} \times \mathrm{S} \times \mathrm{S} / 2$ where $\mathrm{V}$, tumor volume; $\mathrm{L}$, long diameter; and $\mathrm{S}$, short diameter). For TUNEL staining, the primary tumors were removed from the nude mice and subsequently snap-frozen in liquid nitrogen. The frozen sections $(7-\mathrm{mm})$ were then treated with $4 \%$ paraformaldehyde for $30 \mathrm{~min}$ and incubated with $0.3 \% \mathrm{H}_{2} \mathrm{O}_{2}$ for $20 \mathrm{~min}$. The sections were then permeabilized with $0.1 \%$ Triton $\mathrm{X}-100$ for $2 \mathrm{~min}$ and dyed with TUNEL reaction mixture $(5 \mu \mathrm{TdT}+45 \mu 1$ fluorescein-dUTP) for $60 \mathrm{~min}$. Images were immediately captured using fluorescence microscopy.

Statistical analysis. Statistical analysis was performed using SPSS 17.0 software (SPSS Inc., Chicago, IL, USA). The data are expressed as the mean \pm standard deviation. Comparison of $>2$ groups was performed using one-way analysis of variance with Holm-Sidak's post hoc multiple comparison test, and comparison between two groups was performed with two-tailed Student's t-test. $\mathrm{P}<0.05$ was considered to indicate a statistically significant difference.

\section{Results}

Genipin disrupts cell viability and leads to morphological changes in HCT116 cells. Genipin has shown the ability to disrupt growth in several cancer cell lines, including human gastric cancer AGS cells (7), human hepatocellular carcinoma Hep3B cells (8) and human breast cancer MDA-MB-231 cells (9). However, to the best of our knowledge, genipin has not yet been investigated in colon cancer cells.

To evaluate the effect of genipin on colon cancer cells, HCT116 and SW480 cells were treated with different concentrations of genipin and cell viability was determined. The results demonstrated that genipin was able to disrupt the viability in both HCT116 and SW480 cells in a dose- and time-dependent manner (Fig. 1A and B). Furthermore, the $\mathrm{IC}_{50}$ values were calculated, with an $\mathrm{IC}_{50}$ value of $463.4 \mu \mathrm{M}$ at $12 \mathrm{~h}$, $377.6 \mu \mathrm{M}$ at $24 \mathrm{~h}$, and $168.4 \mu \mathrm{M}$ at $36 \mathrm{~h}$. Based on $\mathrm{IC}_{50}$ calculations, further experiments were performed at concentrations of 200, 400 and $600 \mu \mathrm{M}$ genipin for $24 \mathrm{~h}$. 

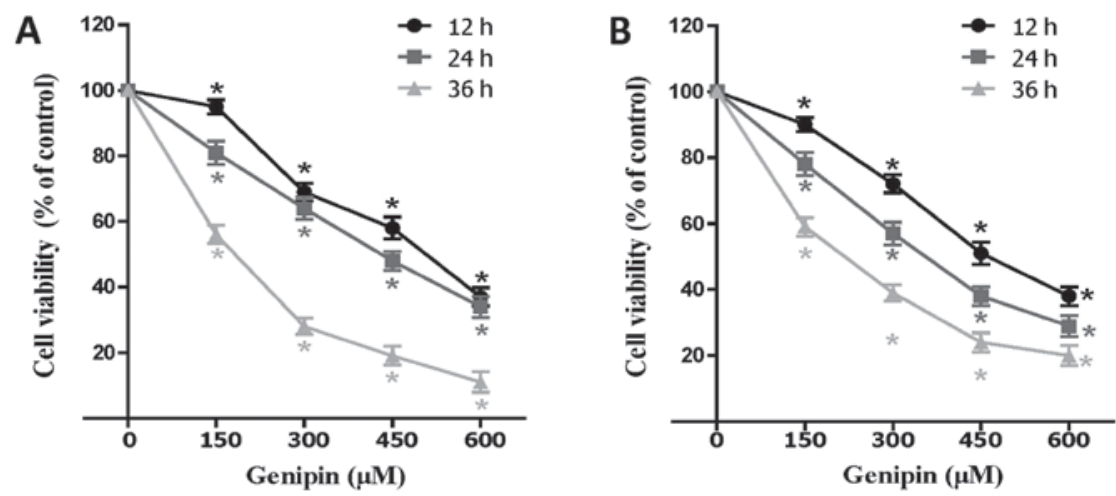

Figure 1. Effect of genipin on the viability of cultured colon cancer cells. The viability of (A) HCT116 and (B) SW480 cells was evaluated by CCK- 8 assay following treatment with $0.1 \%$ DMSO and different concentrations of genipin for 12,24 and $36 \mathrm{~h}$. Relative cell viability was compared with the $0.1 \%$ DMSO-treated groups; $\mathrm{n}=3$. Data are expressed as the mean \pm standard deviation. $\mathrm{P}<0.05$ vs. 0 h. CCK- 8 , Cell Counting kit- 8 ; DMSO, dimethyl sulfoxide.

A

AF

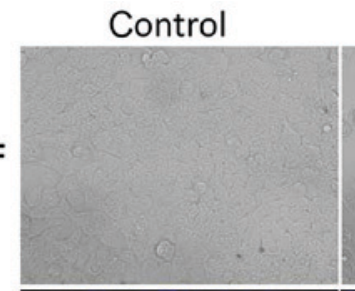

DAPI

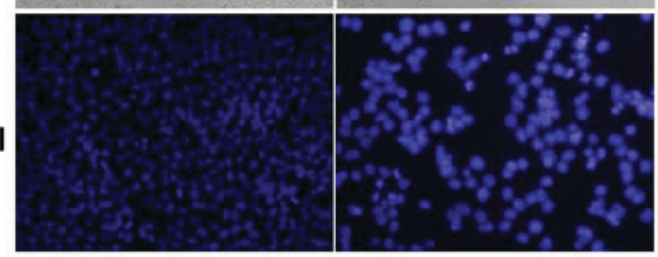

B

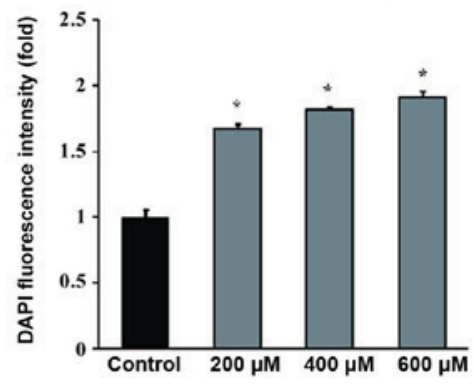

$200 \mu \mathrm{M}$
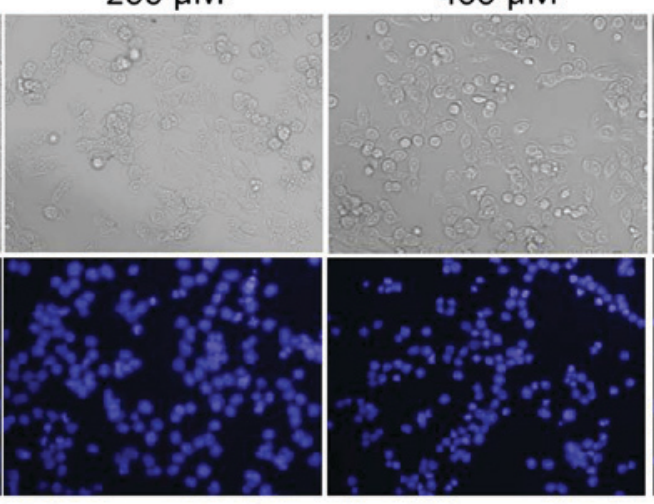

$400 \mu \mathrm{M}$
$600 \mu \mathrm{M}$

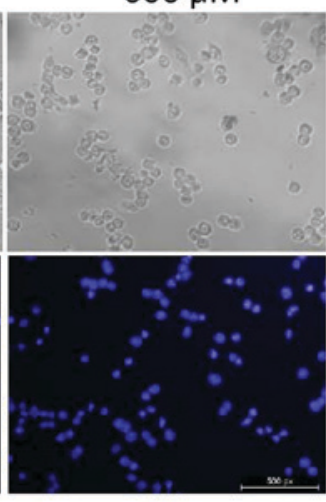

Figure 2. Effect of genipin on the morphological characteristics of HCT116 cells. (A) The morphological changes and DAPI staining of HCT116 cells treated with $0.1 \%$ DMSO and 200, 400 and $600 \mu \mathrm{M}$ genipin were observed using fluorescence microscopy. Scale bar, $25 \mu \mathrm{m}$; magnification, x 200 . (B) The mean fluorescence intensity was calculated by Image-ProPlus 6.0 software; $n=3$. Data are expressed as the mean \pm standard deviation. " $P<0.05$ vs. control. DMSO, dimethyl sulfoxide.

In order to investigate the effect of genipin on the morphological characteristics of colon cancer cells, DAPI staining was used in HCT116 cells treated with genipin, and it revealed that a higher percentage of genipin-treated HCT116 cells exhibited the typical morphological changes of apoptosis, such as cell atrophy, membrane bubbling, DNA fragmentation and nucleus shrinkage under the microscope (Fig. 2A). The fluorescence intensity of DAPI increased with the increase in the dose of genipin (Fig. 2A and B), indicating a higher percentage of apoptotic cells. These results suggested that genipin restrained the growth of HCT116 cells and increased the cell death rate in a dose-dependent manner.

Genipin induces G0/G1 phase arrest and apoptosis in HCT116 cells. To further elucidate the mechanisms of genipin-induced disruption of cell growth, cell cycle and apoptosis analysis were performed in HCT116 cells. Cell cycle analysis revealed that the percentage of cells in the G0/G1 phase increased from $40.79 \%$ (control) to $50.55 \%$ $(200 \mu \mathrm{M})$ and $56.63 \%(300 \mu \mathrm{M})$, whereas the percentage of cells in the $\mathrm{S}$ phase decreased with the corresponding treatments (Fig. 3A and B). The disruption in the cell cycle appeared to correspond to a higher percentage of apoptotic cells among genipin-treated cells (13.1\% for $200 \mu \mathrm{M}$ and $19.7 \%$ for $300 \mu \mathrm{M}$ ) compared with the control group (2.8\%) (Fig. 3C and D). These results were consistent with previous reports demonstrating that genipin significantly disrupted cell cycle progression in rat C6 glioma cells (11) and human leukemia K562 cells (12), and promoted apoptosis in rat C6 glioma cells (11). 

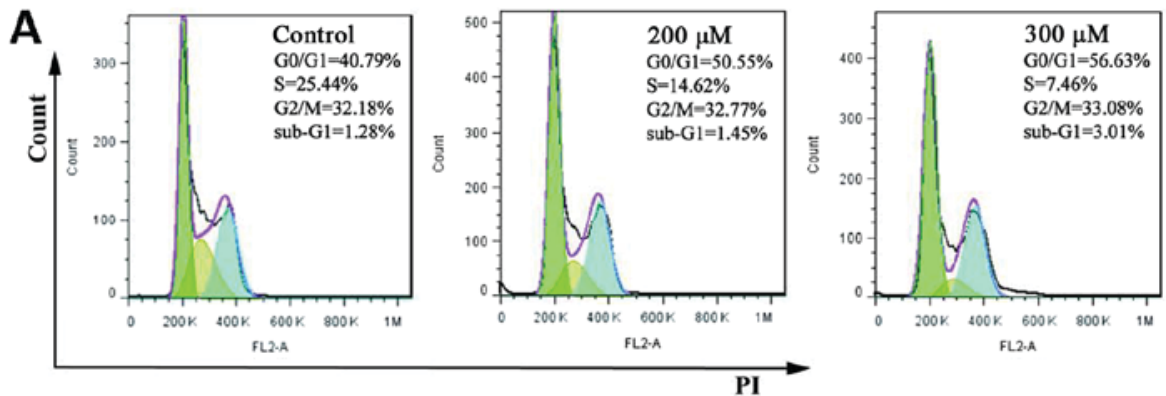

C

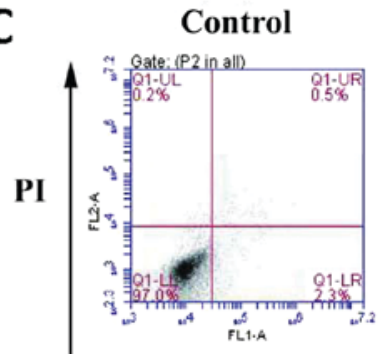

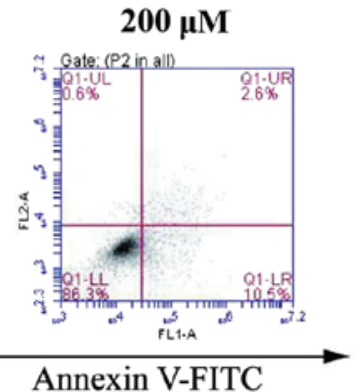

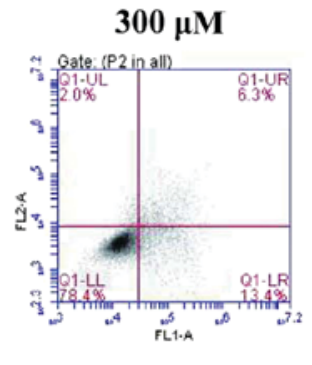

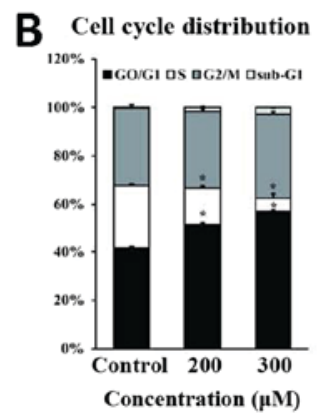

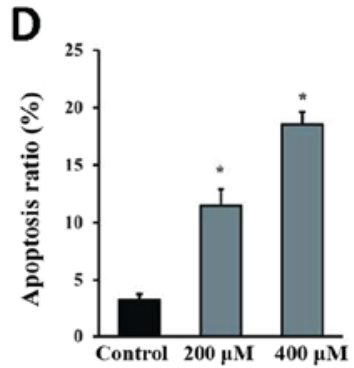

Figure 3. Effect of genipin on cell cycle distribution and apoptosis of HCT116 cells. The cell cycle distribution and apoptosis of HCT116 cells were analysed by flow cytometry and the data were obtained from 10,000 events. (A) Cell cycle distribution was analyzed by PI staining and determined using FlowJo 7.6 software. (B) Histogram of cell cycle distribution; $n=3$. (C) Apoptosis was analyzed with FITC-Annexin V and PI staining. (D) The percentages of apoptotic cells are shown in a histogram; $n=3$. Data are expressed as the mean \pm standard deviation. ${ }^{*} \mathrm{P}<0.05$ vs. control. PI, propidium iodide; FITC, fluorescein isothiocyanate.

Genipin reduces MMP and increases ROS generation in HCT116 cells. ROS generation plays a crucial role in mediating apoptosis induction. The increase in ROS may lead to loss of MMP and increase oxidative cell damage, ultimately contributing to apoptosis (13). Based on this understanding, MMP and ROS changes in HCT116 cells were evaluated following genipin treatment, and a dose-dependent decrease in MMP was observed with genipin treatment (Fig. 4A and B). Intracellular ROS production was also measured and, as illustrated in Fig. 4C and D, genipin treatment significantly increased the intracellular ROS level in HCT116 cells. Collectively, these results indicate that ROS generation and mitochondrial dysfunction are involved in genipin-induced apoptosis in HCT116 cells. These results are consistent with recent reports demonstrating that genipin significantly interferes with the function of uncoupling protein 2, ultimately disrupting the protein gradient across the inner mitochondrial membrane and increasing ROS production (14). In addition, the ROS-antagonizing agent $\mathrm{N}$-acetyl-L-cysteine (NAC) blunted the disruptive effect of genipin on the viability in HCT116 cells (Fig. 4E), indicating that genipin-induced disruption of cell viability may be dependent on ROS generation.

Genipin regulates the expression of apoptosis-associated proteins in HCT116 cells. The balance between the anti-apoptotic gene $\mathrm{Bcl}-2$ and the pro-apoptotic gene Bax plays a key role in cellular homeostasis, and abnormal Bax/Bcl-2 ratio may cause apoptosis by altering the MMP (15). Caspase-3 is regulated by multiple genes associated with apoptosis and is considered as the most important terminal cutting enzyme in the apoptotic process; an increase in cleaved caspase-3 levels plays a crucial role in apoptosis initiation (16). In addition, Bcl-2 family proteins and caspase-3 are key regulatory factors in the mitochondrial-mediated apoptotic induction.
To elucidate whether the mitochondrial apoptotic pathway is involved in genipin-induced apoptosis in HCT116 cells, the protein expression of Bcl-2, Bax and cleaved caspase-3 were evaluated by western blotting (Fig. 5). There was a significant upregulation in the protein expression of pro-apoptotic Bax and cleaved caspase- 3 following genipin treatment, while the expression of anti-apoptotic Bcl-2 was noticeably decreased (Fig. 5A and B). Thus, genipin may promote cell death through Bax-dependent activation of the mitochondrial pathway in HCT116 cells.

The tumor suppressor p53 regulates cell cycle arrest, apoptosis and the DNA repair process. In the present study, genipin treatment significantly increased the expression of the p53 protein (Fig. 5A and B), suggesting that p53 may play an important role in genipin-induced apoptosis. Upregulation of p53 may also explain the cell cycle arrest induced by genipin, due to the canonical ability of p53to activate p21 expression and cause G1 arrest (17).

Genipin causes significant tumor growth inhibition and apoptosis in nude mice. To verify the effect of genipin on HCT116 cells in vivo, nude mice were subcutaneously xenografted with HCT116 and SW480 cells, and were then treated with 20,40 and $80 \mathrm{mg} / \mathrm{kg}$ of genipin. Based on tumor volume measurements, genipin exerted an inhibitory effect on HCT116 tumor growth as indicated by a decrease in tumor volume by $33 \%(20 \mathrm{mg} / \mathrm{kg})$, $57 \%(40 \mathrm{mg} / \mathrm{kg})$ and $73 \%(80 \mathrm{mg} / \mathrm{kg})$ compared with the vehicle control group (Fig. 6A and B). Furthermore, a similar inhibitory effect of genipin on tumor growth was observed when nude mice were subcutaneously xenografted with SW480 cells (Fig. 6C and D). Western blotting analysis revealed that genipin increased the expression of $\mathrm{p} 53, \mathrm{Bax}$ and cleaved caspase-3, and reduced the expression of Bcl-2 (Fig. 6E and F), 
A
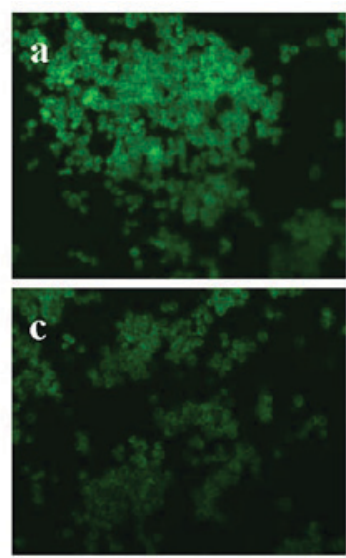

C

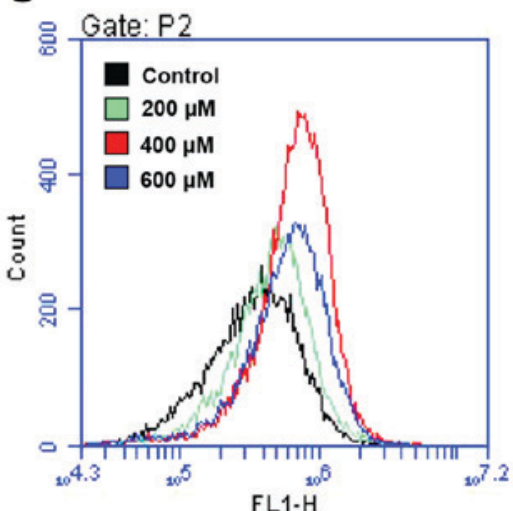

B

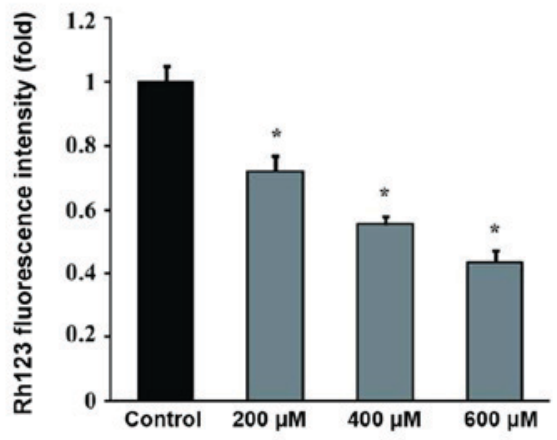

E

Figure 4. Effect of genipin on MMP and ROS. (A) MMP was detected by Rhodamine 123 staining after cells were treated with (a) $0.1 \%$ DMSO, (b) $200 \mu \mathrm{M}$, (c) $400 \mu \mathrm{M}$ and (d) $600 \mu \mathrm{M}$ genipin. Scale bar, $25 \mu \mathrm{m}$; magnification, x200. (B) Mean fluorescence intensity was calculated by Image-ProPlus 6.0 software; $n=3$. (C) ROS production was detected by flow cytometric analysis after DCFH-DA staining. (D) The generation of ROS is shown in a histogram; $\mathrm{n}=3$. (E) Effect of NAC on the inhibitory effect of genipin on the viability of HCT116 cells, $\mathrm{n}=5$. Data are expressed as the mean \pm standard deviation ${ }^{*} \mathrm{P}<0.05$ vs. control. ${ }^{\text {P }}<0.05$ vs. genipin. MMP, mitochondrial membrane potential; ROS, reactive oxygen species; DMSO, dimethyl sulfoxide; DCFH-DA, dichloro-dihydro-fluorescein diacetate; NAC, N-acetyl-L-cysteine.

A

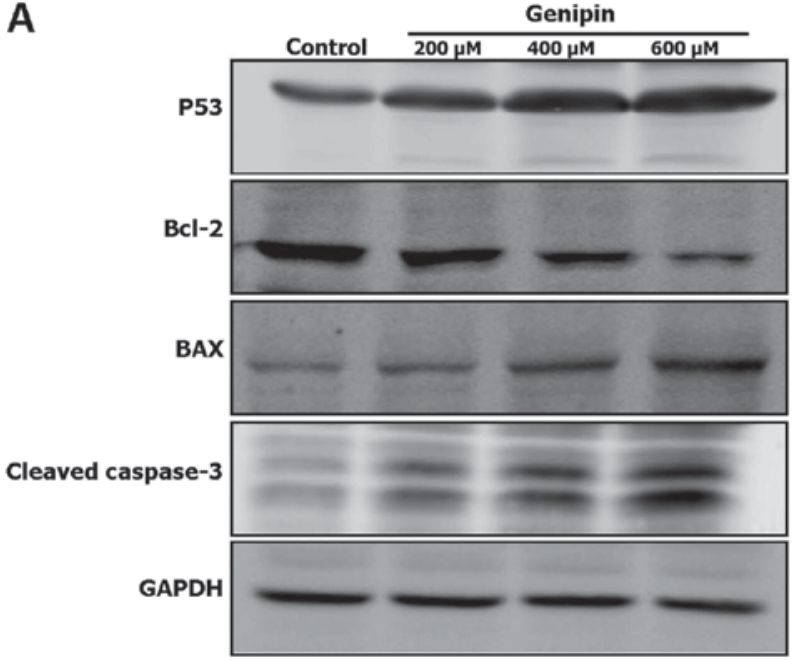

B

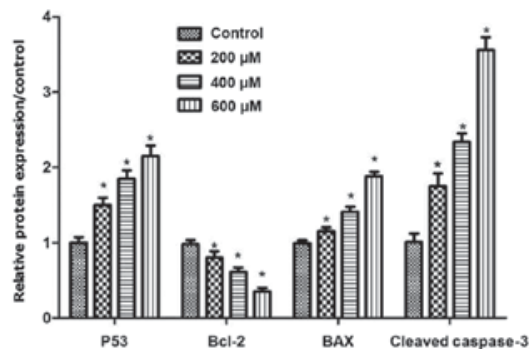

Figure 5. Effect of genipin on the expression of apoptosis-associated proteins in colon cancer cells. (A) The expression levels of apoptosis-associated proteins in HCT116 cells treated with genipin $(0,200,400$ and $600 \mu \mathrm{M})$ for $24 \mathrm{~h}$ were analyzed by western blotting using anti-p53, anti-Bcl-2, anti-Bax and anti-cleaved caspase- 3 antibodies. (B) The results of the western blot analysis were quantified; $\mathrm{n}=5$. Data are expressed as the mean \pm standard deviation. ${ }^{*} \mathrm{P}<0.05$ vs. control. Bcl, B-cell lymphoma.

consistently with the results of in vitro experiments (Fig. 5). TUNEL staining of tumor sections demonstrated that apoptosis gradually increased with the increase in genipin concentration (Fig. 6G and H). Taken together, these results suggest that genipin exerted a significant antitumor effect on colon tumor xenografts in nude mice, and this antitumor effect 
A

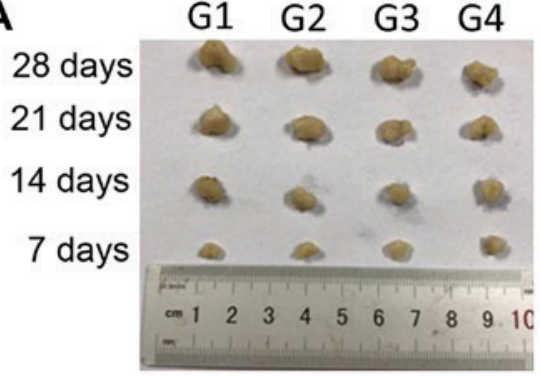

C

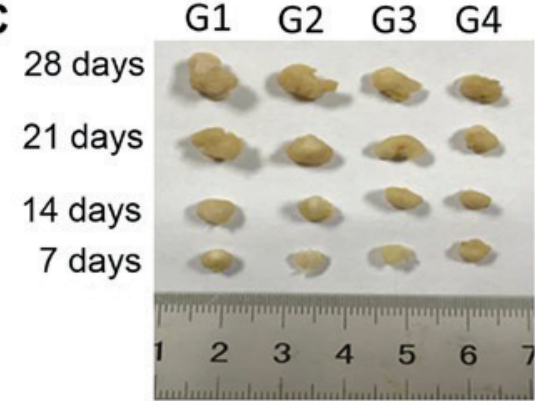

E

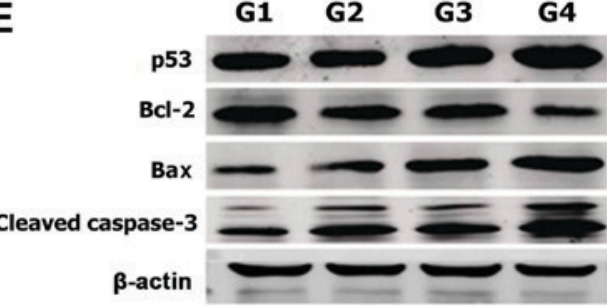

G

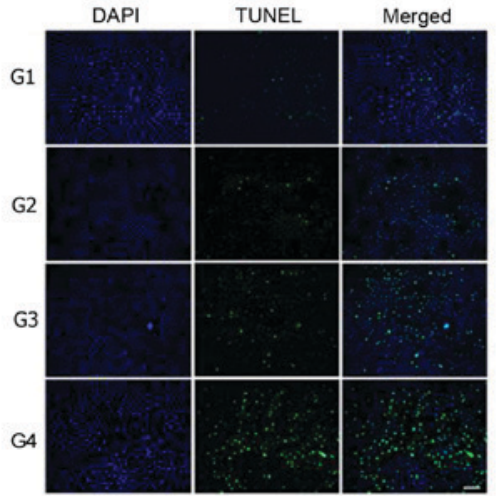

B

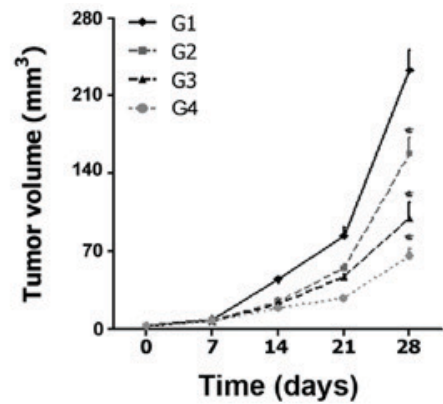

D

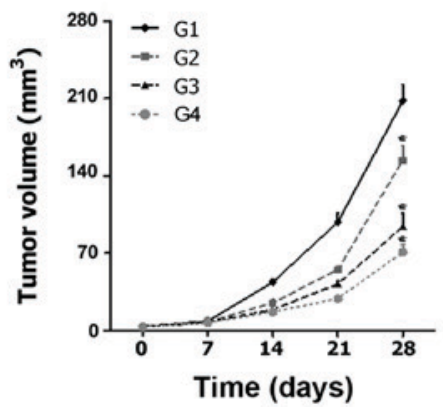

$\mathbf{F}$

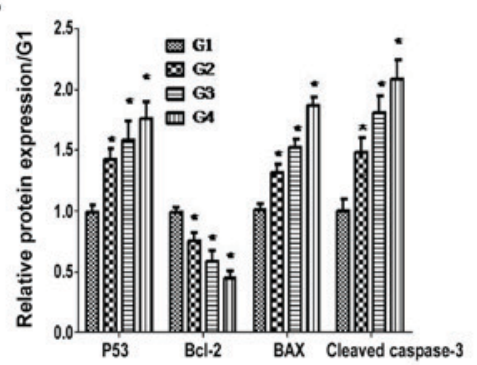

H

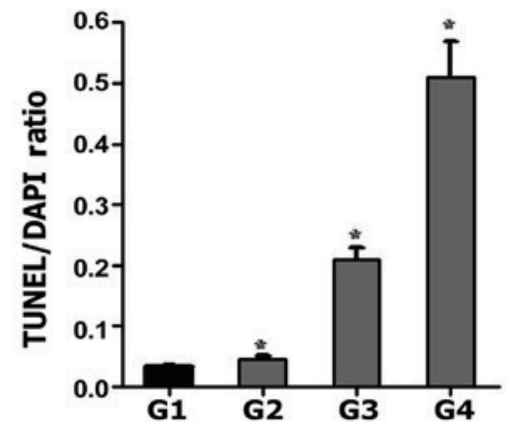

Figure 6. Antitumor effect of genipin on colon cancer cells in vivo. Tumor formation in nude mice injected with HCT116 and SW480 cells was evaluated after the mice were exposed to 20,40 and $80 \mathrm{mg} / \mathrm{kg}$ (G2, G3 and G4, respectively) genipin, while the control group was gavaged with saline (G1) for 28 days . (A) Tumor formation and (B) tumor diameter in nude mice after HCT116 cell injection on the day of harvest. (C) Tumor formation and (D) tumor diameter in nude mice after SW480 cell injection on the day of harvest. The diameter of the tumors was measured by vernier calipers: Tumor volume=long diameter $\mathrm{x}$ short diameter $\mathrm{x}$ (short diameter/2); $\mathrm{n}=5$. (E) Protein expression was analyzed by western blotting and the results were (F) quantified. (G) Frozen sections were stained with TUNEL and examined using fluorescence microscopy; scale bar, $50 \mu \mathrm{m}$; magnification, $\mathrm{x} 100$. (H) The percentages of apoptotic cells are shown in a histogram; $\mathrm{n}=5$. Data are expressed as the mean \pm standard deviation. ${ }^{*} \mathrm{P}<0.05$ vs. G1.

maybe associated with p53 and Bax-mediated activation of the mitochondrial apoptosis pathway.

\section{Discussion}

Genipin arrests the growth of several types of cancer cells, such as AGS human gastric cancer cells (7), Hep3B human hepatocellular carcinoma cells (8) and MDA-MB-231 human breast cancer cells (9). However, the effect of genipin on colon cancer cells remains unclear. In the present study, genipin exerted a dose-dependent antitumor effect on HCT116 and SW480 cells in vitro and in vivo, which may be attributed to genipin-induced G0/G1 cell cycle arrest and apoptosis. It has been reported that genipin not only significantly increased the G0/G1 ratio of rat C6 glioma cells (11), but also markedly inhibited G2/M phase transition in human leukemia K562 
cells (12). In this experiment, genipin arrested cell cycle progression in the G0/G1 phase in HCT116 cells, which may be associated with activation of p53.

The tumor suppressor $\mathrm{p} 53$ performs important functions in regulating cell growth and cell death: It participates in the cell cycle checkpoint pathway, which allows cells to repair both endogenous and exogenous DNA damage; it also regulates multiple genes, including p21, which is associated with G1 phase arrest (17). Similarly, the results of the present study revealed that genipin treatment significantly increased the expression of the p53 protein in vivo and in vitro.

ROS lead to MMP loss and oxidative cell damage, eventually contributing to apoptosis (13). This study suggested that genipin promoted cell death via the generation of ROS and the reduction of MMP. Recent research demonstrated that genipin significantly interferes with the function of uncoupling protein 2 , which dissipates the proton gradient across the inner membrane of the mitochondria and decreases ROS production (14). Furthermore, ROS-antagonizing agents, such as NAC, blunted the disruptive effect of genipin on the viability of HCT116 cells, indicating that genipin-induced disruption in cell viability may be dependent on ROS generation.

The balance between the anti-apoptotic gene $\mathrm{Bcl}-2$ and the pro-apoptotic gene Bax plays a key role in cell development. Abnormal expression of $\mathrm{Bax}$ and $\mathrm{Bcl}-2$ triggers apoptosis via the mitochondrial pathway (15). Caspase- 3 is regulated by multiple genes associated with apoptosis and is considered as the most important terminal cutting enzyme in the apoptotic process (16). Additionally, Bcl-2 family proteins and caspase- 3 are key regulatory factors of the mitochondrial-mediated apoptosis pathway. In the present study, the levels of Bax and cleaved caspase- 3 were markedly upregulated, while the expression of Bcl-2 decreased significantly following treatment with genipin, demonstrating that genipin promoted apoptosis via the Bax-initiated mitochondrial-mediated pathway.

In conclusion, genipin exerted a dose-dependent inhibitory effect on the growth of HCT116 and SW480 cells. The inhibitory mechanism was associated with cell cycle arrest at the G0/G1 phase by induction of the expression of p53. Genipin also induced ROS generation and MMP decrease, and finally triggered apoptosis by upregulating the expression of $\mathrm{Bcl}-2$ family proteins and activating caspase-3. Taken together, these findings demonstrated that genipin suppressed the proliferation and enhanced the apoptosis of colon cancer cells; thus, it may prove useful as a novel drug for the prevention and treatment of colon cancer. However, the detailed molecular mechanism remains unknown and further investigation is required to elucidate it.

\section{Acknowledgements}

Not applicable.

\section{Funding}

The present study was supported by grants from the Science and Technology program of Chongqing (grant no. cstc2013yykfB10006) and the ' 111 Project' for Biomechanics and Tissue Repair Engineering, China (grant no. 32450183).

\section{Availability of data and materials}

The datasets used during the present study are available from the corresponding author on reasonable request.

\section{Authors' contributions}

XW and LL conceived the project and designed the experiments. JY and JL conducted the experiments. JY wrote the manuscript. XW and LL revised the manuscript. All authors have reviewed and approved the final version of this manuscript.

\section{Ethics approval and consent to participate}

The present study was approved by the Third Military Medical University Animal Use and Care Committee.

\section{Patient consent for publication}

Not applicable.

\section{Competing interests}

The authors declare that they have no competing interests.

\section{References}

1. Siegel RL, Miller KD, Fedewa SA, Ahnen DJ, Meester RGS, Barzi A and Jemal A: Colorectal cancer statistics, 2017. CA Cancer J Clin 67: 177-193, 2017.

2. Jeyamohan S, Moorthy RK, Kannan MK and Arockiam AJ: Parthenolide induces apoptosis and autophagy through the suppression of PI3k/AKT signaling pathway in cervical cancer. Biotechnol Lett 38: 1251-1260, 2016.

3. Ramezanpour M, Da SK and Sanderson BJ: Venom present in sea anemone (heteractis magnifica) induces apoptosis in non-small-cell lung cancer A549 cells through activation of mitochondria-mediated pathway. Biotechnol Lett 36: 489-495, 2014.

4. Koo HJ, Lim KH, Jung HJ and Park EH: Anti-inflammatory evaluation of gardenia extract, geniposide and genipin. J Ethnopharmacol 103: 496-500, 2006.

5. Park EH, Joo MH, Kim SH and Lim CJ: Antiangiogenic activity of gardenia jasminoides fruit. Phytother Res 17: 961-962, 2003.

6. Suzuki Y, Kondo K, Ikeda Y and Umemura K: Antithrombotic effect of geniposide and genipin in the mouse thrombosis model. Planta Medica 67: 807-810, 2001.

7. Kim JM, Ko H, Kim SJ, Shim SH, Ha CH and Chang HI: Chemopreventive properties of genipin on ags cell line via induction of JNK/Nrf2/are signaling pathway. J Biochem Mol Toxicol 30: 45-54, 2016.

8. Kim BC, Kim HG, Lee SA, Lim S, Park EH, Kim SJ and Lim CJ: Genipin-induced apoptosis in hepatoma cells is mediated by reactive oxygen species/c-JUN NH2-terminal kinase-dependent activation of mitochondrial pathway. Biochem Pharmacol 70: 1398-1407, 2005.

9. Kim ES, Jeong CS and Moon A: Genipin, a constituent of gardenia jasminoides ellis, induces apoptosis and inhibits invasion in MDA-MB-231 breast cancer cells. Oncol Rep 27: 567-572, 2012.

10. Cao HL, Feng QA, Xu W, Li X, Kang Z, Ren Y and Du L: Genipin induced apoptosis associated with activation of the c-JUN NH2-terminal kinase and p53 protein in hela cells. Biol Pharm Bull 33: 1343-1348, 2010.

11. Chang YC, Chou FP, Huang HP, Hsu JD and Wang CJ: Inhibition of cell cycle progression by penta-acetyl geniposide in rat c6 glioma cells. Toxicol Appl Pharmacol 198: 11-20, 2004.

12. Feng Q, Cao, Hl Xu W, Li XR, Ren YQ and Du LF: Apoptosis induced by genipin in human leukemia k562 cells: Involvement of c-JUN N-terminal kinase in G(2)/M arrest. Acta Pharmacol Sin 32: 519-527, 2011. 
13. Simon HU, Haj-Yehia A and Levi-Schaffer F: Role of reactive oxygen species (ROS) in apoptosis induction. Apoptosis 5: 415-418, 2000.

14. Cho YS, Lee JH, Jung KH, Park JW, Moon SH, Choe YS and Lee KH: Molecular mechanism of (18) F-FDG uptake reduction induced by genipin in T47D cancer cell and role of uncoupling protein-2 in cancer cell glucose metabolism. Nucl Med Biol 43: $587-592,2016$

15. Kuwana T, Mackey MR, Perkins G, Ellisman MH, Latterich M, Schneiter R, Green DR and Newmeyer DD: Bid, bax and lipids cooperate to form supramolecular openings in the outer mitochondrial membrane. Cell 111: 331-342, 2002.
16. Yun N, Kim C, Cha H, Park WJ, Shibayama H, Park IS and Oh YJ: Caspase-3-mediated cleavage of picot in apoptosis. Biochem Biophys Res Commun 432: 533-538, 2013.

17. Jayaraman L and Prives C: Covalent and noncovalent modifiers of the p53 protein. Cell Mol Life Sci 55: 76-87, 1999.

(i) (3) This work is licensed under a Creative Commons Attribution-NonCommercial-NoDerivatives 4.0 International (CC BY-NC-ND 4.0) License. 